\title{
ZIGBEE SENSOR NETWORK INTEGRATED WITH 4G FOR IOT APPLICATIONS
}

\author{
K. Kalaivani ${ }^{1}$, R. Aravindh ${ }^{2}$, G. Vijayalakshmy ${ }^{3}$ \\ ${ }^{I}$ Student, ECE, Perunthalaivar Kamarajar Institute of Engineering and Technlogy, India \\ ${ }^{2}$ Student, ECE, Perunthalaivar Kamarajar Institute of Engineering and Technlogy, India \\ ${ }^{3}$ Assistant Professor, Electronics and Communication Engineering, Perunthalaivar Kamarajar Institute of Engineering \\ and Technology, India
}

\begin{abstract}
Internet of Things (IoT) refers to uniquely identifiable objects and their virtual representations of an Internet like structures. The main goal of the IoT is to make the sense the information of the computing device without the need of human interaction. Zigbee Wireless sensor node is a communication protocol which is used to transmit data over long distances by passing data through the intermediate devices to cover longer distant and provides secure networking. It can be used anywhere at any time. It requires less power to operate and it can be implemented easily with any type of wireless generation. $4 G$ (Fourth Generation) is the next generation of wireless networks and it can provide fast and secure data transfer. Speed of $4 G$ can be achieved by OFDM modulation and it will utilize most of the existing wireless communication infrastructure. World Wide Interoperability for Microwave Access (WiMAX) is wireless technology and it provides wireless data over long distance. WiMAX provides a media access control (MAC) and proposes the full range of security features to secured data exchange. It transmits the data at $70 \mathrm{Mbps}$. In this paper, Zigbee Wireless sensor nodes real time data transmission integrated with $4 G$ Technology for long distance Secure Communication with high mobility is proposed and analyzed using OPNET. The performance such as delay, data sent, Jitter and end-to-end delay and packet delay variation are simulated.
\end{abstract}

Keywords-WiMAX, 4G, IoT, Zigbee, Wireless, sensor network, voice

\section{INTRODUCTION}

Two different technologies which find a swift and a massive growth at present are Cloud Computing and the next one is Internet of Things (IoT). The next era of computing will be out of the present traditional computing, where the computers will be able to access the data without any human interaction on the field. Internet of Things (IoT) represents an integral part of the future internet [1]. IoT can be realized into three paradigms as IP (Internet Protocol) oriented (middleware), things oriented (sensors) and semantic-oriented (knowledge) [2]. Zigbee is the Wireless sensor standard based technology that addresses the unique needs of most remote applications [3]. Zigbee networks provide security using 128 bit symmetric encryption keys and uses Direct Sequence Spread Spectrum (DSSS) [4]. It supports for multiple network topologies such as point-to-point, point-tomultipoint and mesh networks [5]. 4G is a technology which supports high quality audio and video streaming end to end IP [6]. 4G system targets peak data rates of approximately 100 Mpbs for high mobility and $1 \mathrm{Gbps}$ for low mobility services. It supports bandwidth in a range of 5 to $20 \mathrm{MHz} 4 \mathrm{G}$ supports both the TDD and FDD duplexing [7]. It uses orthogonal frequency division multiplexing (OFDM) access for LTE DL, WiMAX UL, WiMAX DL and single carrier frequency division multiple (SC-FDMA) access for LTE UL [8]. WiMAX is an IEEE 802.16 standard which is highly efficient and is suitable for long range applications. WiMAX base station can offer greater wireless coverage of about 5 miles in a line of sight transmission within the bandwidth of up to $70 \mathrm{Mbps}$ [9]. Zigbee module is a wireless sensor network (WSN) platform which is based on the IEEE 802.15.4, 2.4 GHz standard [10]. Zigbee uses two types of commands for communication- (i) AT commands and (ii) API commands. Zigbee can be either used as a coordinator or a router or an end device. It uses serial communication protocol for the transfer of data and operates at a range of 3.3V DC [11]. The coordinator organizes the network and maintains the routing and it responsible for initializing, maintaining and controlling the network. Router communicates to the coordinators, to other routers and to end devices. It forms network backbone to the end device can communicate to the router and coordinator but not with another end device [12]. PAN is the combination of a single coordinator, one or more routers and end devices. The routers and end devices can communicate with the coordinator within the PAN. To cover large area more number of PAN is formed [13]. Worldwide Interoperability for Microwave Access (WIMAX) is a $4 \mathrm{G}$ technology used to transmit the wireless data to cover longer distances [14]. WiMAX forum mission is to promote and certify compatibility and interoperability of broadband wireless products [15]. WiMAX offers alternative to the traditional wireless data transmission over the cables and digital subscriber lines. WiMAX is based on the 
radio frequency $(\mathrm{RF})$ technology called Orthogonal Frequency Division Multiplexing (OFDM) [16]. It is an effective way for transferring the data more than carrier width of $5 \mathrm{MHz}$ and below the carrier width of $5 \mathrm{MHz}$ CDMA based $3 \mathrm{G}$ system is sufficient [17]. WiMAX is a standard-based wireless technology and offers high throughput broadband connections. WiMAX provides WMAN connectivity with a speed of $70 \mathrm{Mbps}$ and its base station covers an average distance of 5 to $10 \mathrm{~km} \mathrm{[18].} \mathrm{It}$ provides communication path between a subscriber site and a core network. In this paper Zigbee wireless sensor is integrated with WiMAX technology for data transmission. Here multimedia transmission is considered and analyzed. The scenarios and challenges of next generation wireless communication is discussed in [19].

The rest of the paper is organized as follows. The section II discuss about proposed novel Zigbee and WiMAX network Integration for IoT. In Section III Performance analysis of the propose work is discussed. Section IV concludes the paper.

\section{ZIGBEE AND WIMAX NETWORK INTEGRA-}

\section{TION}

\subsection{Motivation and the Proposed System}

In the literature, Zigbee sensor networks are integrated through either GSM (Globle system for Mobile) 2G or GPRS (General Packet radio System) 2.5G for the data transmission. Here data can be transmitted only to a shorter distance and also smaller coverage area with more delay without any security. Here the motivation is to integrate Zigbee sensor nodes with WiMAX Technology to transmit multimedia data for secure transmission. In the proposed model the data can be transmitted to longer distance without much complexity.

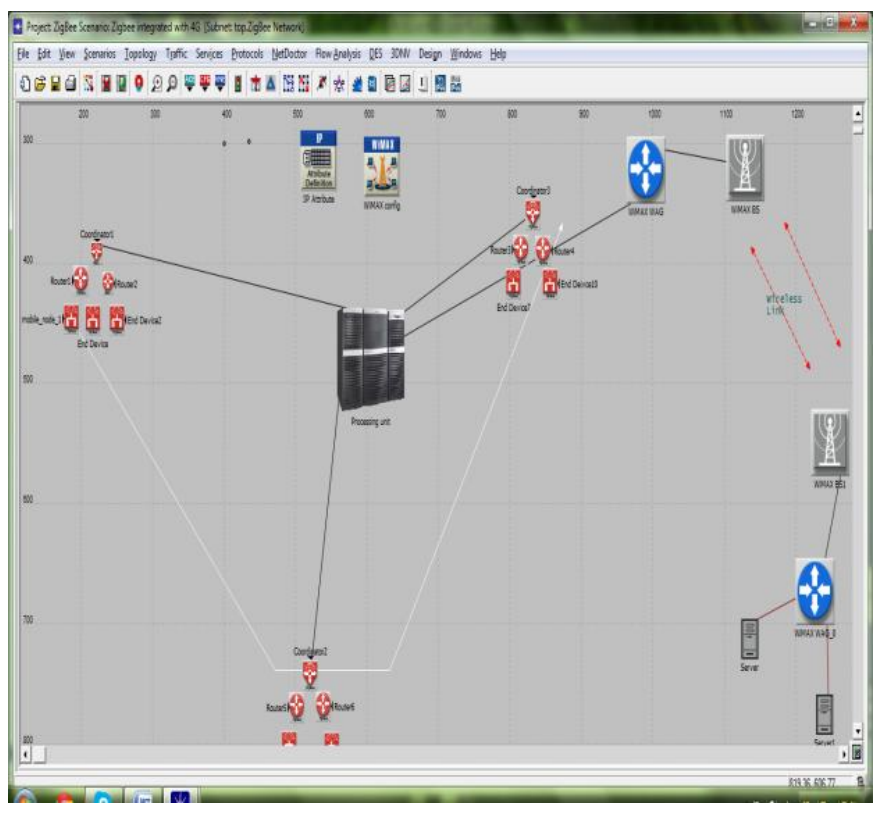

Fig 1 Zigbee module integrated with 4G WiMAX
The Figure 1 shows the proposed architecture of zigbee wireless sensor module integrated with 4G WiMAX for global coverage which is used for the application of Multimedia teleconferencing (Voice \& Video). The network architecture contains three PANs, set to PAN ID 1, 2, and 3. The network also contains a mobile node, initially placed next to PAN 1, with its PAN ID set to Auto-Assigned. The three PANs have their transmit power configured to 2 mill watts so that their coverage areas do not overlap. The trajectory of mobile_node_1 is configured to take the node through the coverage area of each of the three PANs over the course of 20 minutes. Based on this trajectory, it is expected that mobile_node_1 will initially join PAN 1, then switch to PAN 2, and finally to PAN 3. This architecture is designed to cover large area with efficient data traffic. The traffic on each node except mobile_node_1 is configured as Random destination. When they join the network, they will choose a random node within their own PAN and send traffic to that node for the rest of the simulation. Here mobile_node_1 is configured to send traffic to its parent node. Additionally all PAN Zigbee coordinators are interfaced through a processing Controller unit (PCU). This controller is connected to WiMAX base station (BS) through wireless access gateway (WAG). This will act as transmitter part of the proposed architecture. The receiver part is also consists of WiMAX-BS along with WAG where the corresponding application server is present. Initially PAN 1 sends multimedia information with the help of Zigbee Coordinator through end user which is located at the long distance to PAN 2. Then PAN 2 and PAN 3 with the help of Controlling unit which is connected with Zigbee Coordinator communicates 4G Base station and transmit the multimedia information to the receiving $4 \mathrm{G}$ base station and finally Application servers receives the teleconferencing messages which is sent by Zigbee sensor nodes.

\section{PERFORMANCE ANALYSIS}

In Performance analysis teleconferencing, data and Voice applications are analyzed for Zigbee sensor nodes along with 4G network integration for long distance transmission to support high mobility. In $\mathrm{X}$ axis, simulation time period in seconds is taken. In Y axis corresponding parameter is taken. The simulation time period taken for analysis is 2000 seconds.

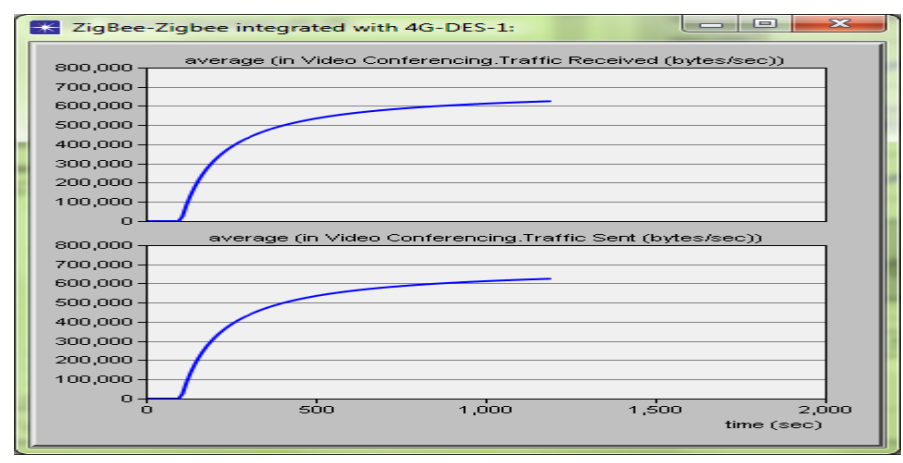

Fig 2 Tele Video Conferencing Traffic received and sent for Zigbee integrated with $4 \mathrm{G}$ 


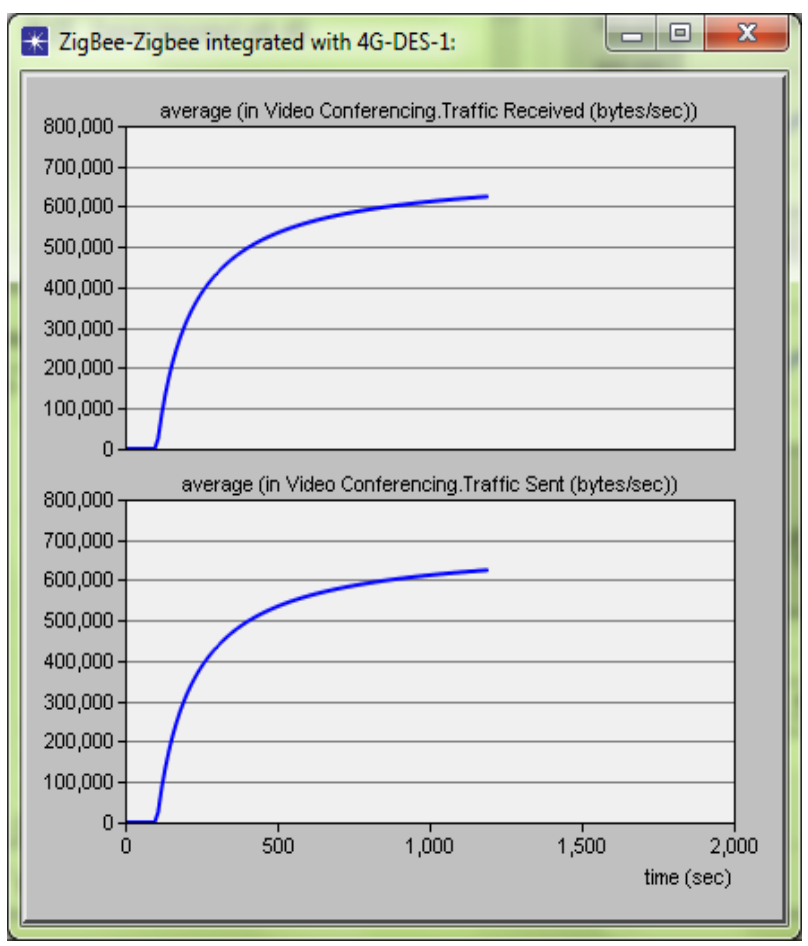

Fig 3 Video Streaming Packet delay variations and packet endto-end delay (sec) for Zigbee integrated with $4 \mathrm{G}$

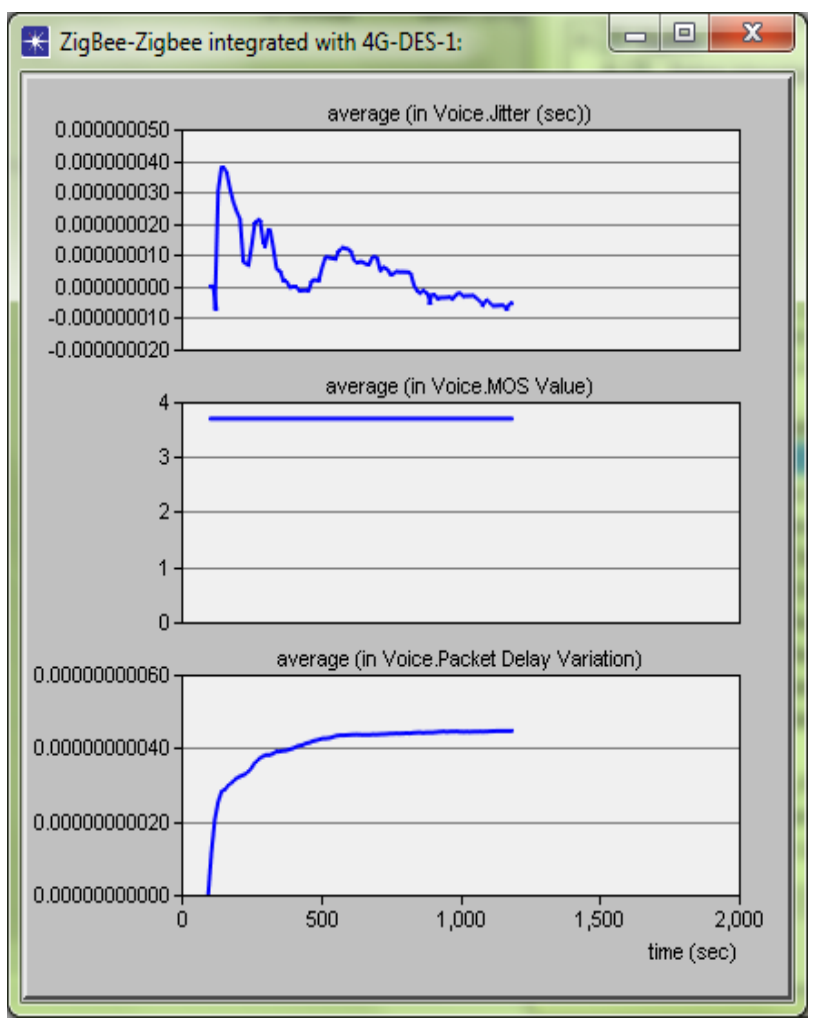

Fig 4 Voice Jitter, Voice MOS (Mean Opinion Score), Voice packet delay Variations for Zigbee integrated with 4G

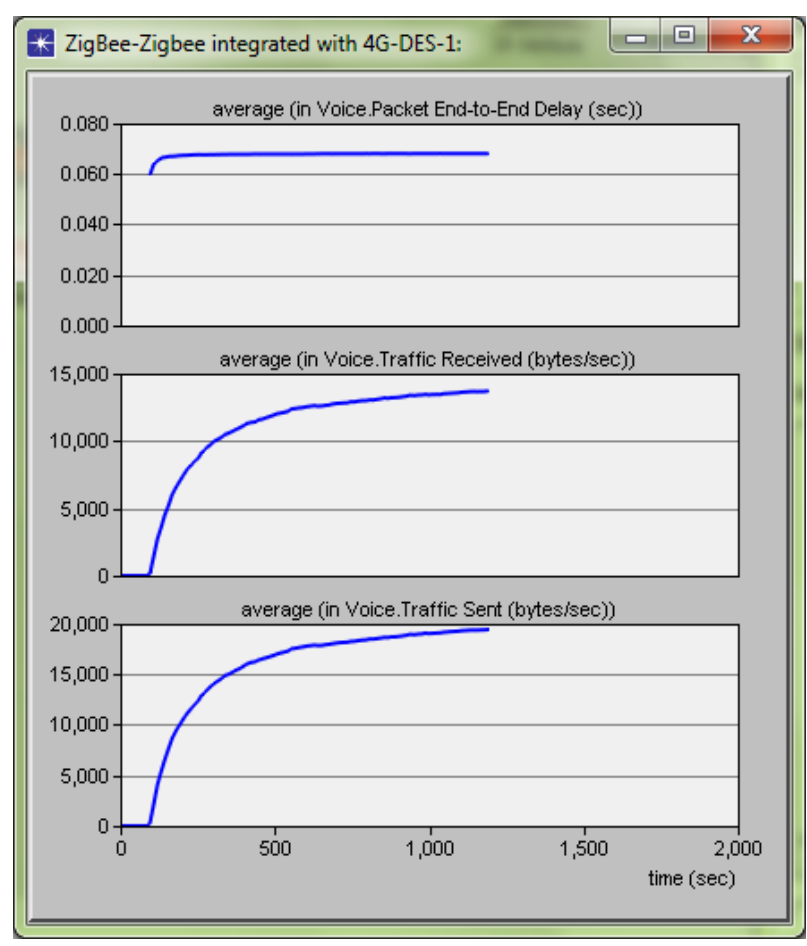

Fig 5 Voice packet end-to-end delay, Voice traffic received and sent for Zigbee integrated with $4 \mathrm{G}$

ZigBee-Zigbee integrated with 4G-DES-1: mobile_nod... \begin{tabular}{ll|l|l|}
\hline & 回 & $X$ \\
\hline
\end{tabular}

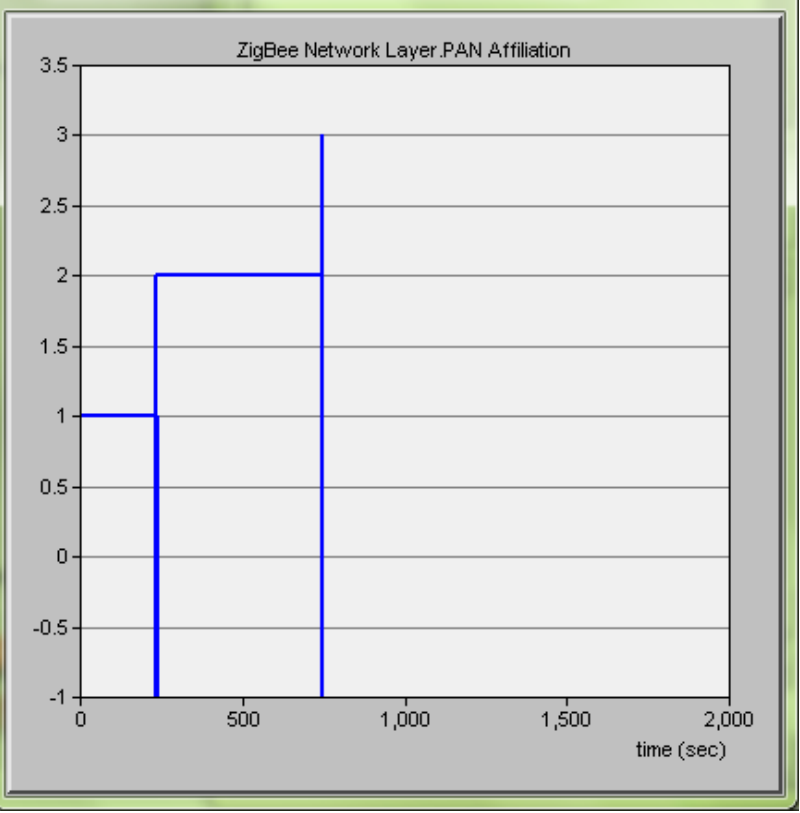

Fig 6 Zigbee Network layer PAN affiliation for for Zigbee integrated with $4 \mathrm{G}$

The figure 2 shows that Video streaming traffic received and sent for the proposed architecture. Here video traffic sent and received is same. This shows that proposed architecture output performs well. This shows there is minimum delay with large 
throughput.The Figure 3 shows that Video Packet delay variations and Packet end-to-end delay for the proposed architecture is very less for the received video traffic of 650,000 bytes per seconds. This also shows global coverage with minimum delay. Since processing unit is connected to all the PAN groups, if any one PAN group fails to transmit data, It identifies and informs to corresponding coordinator to retransmit or otherwise the data copy from other PAN groups can be used to transmit anywhere at any time without waiting for long time. Hence mobile node movement trajectory is captured and sensed by controlling unit and transmit the stored data via $4 \mathrm{G}$ network. In the receiving side again data is received through $3 \mathrm{G} / 4 \mathrm{G}$ using application servers with QoS (Quality of service). From figure 4, Voice Jitter and Voice packet delay variations is also very less. Jitter is nothing but Variation in delay. Here data is sent via all sensor nodes. This shows proposed architecture performs well with very less delay for large traffic. MOS (Mean Opinion Score) value is used to judge the voice quality measurements. The typical MOS value for practical $4 \mathrm{G}$ network is 4 . Here it is 3.9. Voice packet delay variations are also less for the proposed architecture. Here from this figure 5 voice traffic received is more or less same as voice traffic sent. There is only minimum packet loss in the architecture. The voice end-to-end delay is also very less for the architecture. This shows proposed Zigbee sensor Node while integrated with $4 \mathrm{G}$ network, performs well even for real time voice. The figure 6 shows a Pan affiliation of a Zigbee network. PAN Affiliation for mobile_node_1: The graph shows mobile_node_1 joined to PAN 1 for the first 240 seconds of the simulation. The node then briefly joins from the PAN ID 1, and then promptly joins PAN 2. At 720 seconds, the nodes join from PAN 2 and promptly join PAN 3. Finally It collectively sends data to controller unit which in turn connected with $4 \mathrm{G}$ WiMAX.

\section{CONCLUSIONS}

In this paper Wireless sensor Node which is coming under Infrastructure less network, integrated with 4G Technology for data transmission for global coverage especially for security applications in remote areas. The main idea is to reduce the delay of the senor nodes data transmission for large real time multimedia traffic by transmitting through infrastructure architecture like WiMAX network. As a future work The proposed architecture is also connected through 5G, LTE (long term evolution) network as a part of next generation wireless network technology.

\section{REFERENCES}

[1] K. Ashton, That "Internet of Things" thing, RFID Journal (2009).

[2] L. Atzori, A. Iera, G. Morabito, "The Internet of Things: A survey," Computer Networks 54 (2010) 2787-2805.

[3] E. Callaway, P. Gorday, L. Hester, J. Gutierrez, M. Naeve, B. Heile, and V. Bahl, "Home networking with IEEE 802.15.4: a developing standard for low-rate wireless personal area networks," IEEE Communications Magazine, vol. 40, no. 8, pp. 70-77, Aug. 2002.

[4] ZigBee Specification, ZigBee Alliance Std., 2005. [Online]. Available: http://www.zigbee.org

[5] C. Perkins, E. Belding-Royer, and S. Das, "Ad hoc ondemand distance vector (aodv) routing," United States, 2003.

[6] Dr. J. Jayakumari, MIMO-OFDM for " $4 \mathrm{G}$ wireless system," IEEE IJSCT, vol. 2(7), 2010, 2886-2889.

[7] Mauri Rao, "4G wireless technology," NCNTE-2012 AT C.R.I.T., Vashi, Navi Mumbai, Feb. 24-25, 2012.

[8] Hujun Yin and Siavash Alamouti, "OFDMA - A Broadband Wireless Access Technology," IEEE Proc. of Sarnoff Symposium, March 2006.

[9] G. Nair, J. Chou, T. Madejski, K. Perycz, P. Putzolu and J. Sydir, "IEEE 802.16 Medium Access Control and Service Provisioning," Intel Technology Journal, vol. 08, August 2004.

[10] J. S. Lee, "An Experiment on Performance Study of IEEE 802.15.4 Wireless Networks," IEEE, 2005.

[11] Y. Peng, Y. Li, Z. Lu, and J. Yu, "Method for saving energy in Zigbee network," in Proceedings of the 5th International Conference on Wireless Communications, Networking and Mobile Computing (WiCOM '09), pp. 1-3, IEEE, September 2009.

[12] Rathod K, Parikh N, Parikh A, Shah V, "Wireless automation using Zigbee protocols," Ninth International Conference on IEEE, Wireless and Optical Communications Networks (WOCN), 2012.

[13] Wireless Medium Access Control (MAC) and Physical Layer (PHY)

[14] Specifications for Low Rate Wireless Personal Area Networks (WPANs), IEEE Std. 802.15.4, 2003.

[15] Mudit bhalla. \& Anand bhalla, "Generation of mobile wireless technology": A survey, IEEE Trans. (09758887) vol. 5- No.4, August 2010.

[16] U. Varshney, R. Jain, "Issues in emerging 4G wireless networks," Computer, vol. 34, issue. 6, June 2001, pp. $94-96$.

[17] G. Song and Y. Li, "Cross-layer optimization for OFDM wireless networks. Part I:Theoretical framework," IEEE Trans. Wireless Com., vol.4, no. 2, pp. 614-6 24, 2005.

[18] S. Chatterjee, W. A. C Fernando, M. K.. vasantha, "Adaptive modulation based MC-CDMA systems for 4G wireless consumer applications," Consumer Electronics, IEEE Transactions on, vol. 49, issue. 4, Nov. 2003, pp. $995-1003$.

[19] Bria, F. Gessler, O. Queseth, R. Stridh, M. Unbehaun, J. $\mathrm{Wu}$, J. Zander, "4th-generation wireless infrastructures: scenarios and research challenges," Personal Communications, IEEE [see also IEEE Wireless Communications], vol. 8 , issue. 6 , Dec. 2001 , pp. $25-$ 31. 